\title{
Une problématique d'éducation à la santé environnementale au lac Saint-Pierre - Exploration de la dimension socioculturelle
}

Hélène Godmaire et Lucie Sauvé

\section{OpenEdition}

Édition électronique

URL : http://journals.openedition.org/ere/4172

DOI : $10.4000 /$ ere.4172

ISSN : 2561-2271

Éditeur

Centr'ERE

Référence électronique

Hélène Godmaire et Lucie Sauvé, « Une problématique d'éducation à la santé environnementale au lac Saint-Pierre - Exploration de la dimension socioculturelle ", Éducation relative à l'environnement [En ligne], Volume 5 | 2005, mis en ligne le 20 novembre 2005, consulté le 21 février 2020. URL : http:// journals.openedition.org/ere/4172; DOI : 10.4000/ere.4172 


\title{
Une problématique d'éducation à la santé environnementale au lac Saint-Pierre - Exploration de la dimension socioculturelle
}

\author{
Hélène Godmaire et Lucie Sauvé
}

Les auteures remercient le réseau COMERN (subvention du Conseil de recherches en sciences naturelles et en génie du Canada), le Fonds québécois de recherche sur la culture et la société (FQRSC), le ministère de l'Environnement du Québec (subvention Action Environnement) et la Chaire de recherche du Canada en éducation relative à l'environnement de l'Université du Québec à Montréal pour leur soutien financier. Nous tenons également à remercier tous les participants de la région du lac Saint-Pierre.

1 L'ampleur, la multiplication et la complexité croissante des problèmes environnementaux nous amènent à prendre conscience de façon de plus en plus saisissante que la « santé » des écosystèmes est étroitement liée à la santé humaine. La santé environnementale devient une préoccupation sociale majeure, qu'il importe d'aborder dans une perspective écosystémique (Lebel, 2003), voire holistique, tenant compte des multiples dimensions des réalités concernées : biophysiques, écologiques, culturelles, politiques, économiques, etc. Parmi les risques actuels d'atteinte à la santé, ceux qui sont associés à la présence de contaminants dans l'environnement ne peuvent plus être ignorés. La nourriture, l'air, le sol et l'eau contiennent désormais plusieurs types de substances polluantes (métaux lourds comme le mercure, les biphényles polychlorés - BPC, les pesticides, etc.). Ces substances souvent diffuses ont mené à l'apparition de nouveaux types de risques pour la santé humaine et celle des écosystèmes. Il en résulte des atteintes directes et manifestes à la santé et à la qualité de vie (Santé Canada, 1999), mais aussi, de plus en plus d'effets insidieux sont désormais détectés (Villedieu, 2002).

2 Certes, jusqu'à maintenant, les populations à risque ont reçu des recommandations concernant la consommation d'aliments potentiellement contaminés. Toutefois, 
l'histoire de la communication du risque relatif au mercure par exemple montre que des informations ponctuelles, non mises à jour ni contextualisées, parfois même contradictoires, ont souvent plongé les populations dans l'incertitude et le doute. Par ailleurs, malgré l'instauration de politiques gouvernementales visant à réduire les émissions et les rejets, le problème de la présence de contaminants dans l'environnement perdure ; les gestionnaires locaux demeurent souvent à l'écart de la problématique et les communautés touchées restent sans recours formels adéquats.

C'est à une telle problématique globale que s'intéresse le Réseau de recherche intégrée sur le mercure (COMERN $)^{1}$ qui se penche plus spécifiquement sur l'impact de ce métal lourd sur la santé des écosystèmes et celle des êtres humains. Dans le cadre d'une vaste étude pancanadienne entreprise depuis 2001 par COMERN, notre équipe de recherche en éducation relative à l'environnement a reçu un double mandat : clarifier la dimension socioculturelle de la problématique et promouvoir une éducation relative à la santé environnementale au sein des populations concernées. Adoptant une méthodologie d'étude de cas, nous avons surtout centré nos travaux dans la région du lac SaintPierre. Nous avons intégré entre eux les deux volets de notre mandat et entrepris un processus de recherche-intervention collaborative avec différents groupes sociaux de cette région, visant à clarifier leur compréhension, leurs représentations et les perspectives de solution qu'ils envisagent à la problématique de la contamination par le mercure, recadrée d'entrée de jeu dans la problématique plus globale de l'ensemble des contaminants du milieu. La caractérisation des aspects socioculturels de la situation est en effet nécessaire au diagnostic global de la problématique. Cela permet d'enrichir le projet de recherche scientifique d'une dimension psychosociale. Mais plus encore, dans une perspective éthique, il faut reconnaître que le traitement des problématiques environnementales fait appel à une approche participative; dans une perspective stratégique, il importe aussi de favoriser l'appropriation de la "situation problème " par les populations concernées. À cet effet, une démarche d'investigation collaborative peut constituer en elle-même un processus d'éducation communautaire. Or il nous importait de vérifier la contribution potentielle d'une telle stratégie à la mobilisation des groupes sociaux de la région pour participer à un processus collectif de résolution de problèmes, où leurs savoirs, leurs préoccupations, leurs attentes et leurs talents deviennent des éléments essentiels pour la recherche et la mise en place de solutions appropriées.

Cet article témoigne de certains résultats relatifs à la dimension phénoménologique de notre projet, issus de l'exploration collective de la dimension socioculturelle de la problématique. Nous verrons que cette démarche a permis de mettre en évidence une culture régionale bien particulière, ancrée dans l'appartenance au territoire et dans des pratiques ancestrales de chasse et de pêche. Sur cette trame de fond, nous avons pu également saisir et contraster le caractère particulier du rapport au territoire chez différents groupes sociaux de la région. Ces éléments d'un portrait culturel régional fournissent des pistes pour la conception de stratégies d'éducation relative à l'environnement appropriées à chaque population ciblée.

\section{Éléments de contexte}

5 Notre étude de cas collaborative visait donc à saisir certains éléments d'un "système culturel en action » (selon l'expression de Feagin et coll., 1991, in Corcoran et coll., 
2004), en nous penchant sur les représentations et pratiques de différents groupes d'« acteurs sociaux » et en les invitant à échanger entre eux et à se mettre en projet. Puisqu'une étude de cas est essentiellement centrée sur une réalité particulière, dans un contexte particulier, nous présenterons d'abord certaines caractéristiques contextuelles de la région du lac Saint-Pierre.

\section{Contexte historique, géographique et socio-environnemental}

6 Les explorateurs de la Nouvelle France, Cartier et un peu plus tard Champlain, évoquent dans leurs écrits l'extraordinaire richesse de la flore et de la faune, du gibier et du poisson du lac Saint-Pierre (Laverdière, 1870, in De Konink, 2000, p. 3-5) : « L'eau est presque dormante dans le lac qui est fort poissonneux [...] il y a quantité de noyers et de vignes et de belles prairies avec force gibier et animaux sauvages. La pêcherie du poisson y est plus abondante qu'en aucun autre lieu qu'eussions vu ». Le géographe Rodolphe De Konink (2000, p.39) rappelle l'importance historique de l'eau dans la région du lac Saint-Pierre :

Pendant près de deux siècles et demi, la vie aux Cent-îles a été sous le signe de l'eau. Non seulement parce que l'on en tirait des ressources, mais encore et surtout parce l'eau était omniprésente. L'eau et les chenaux ont marqué la mise en valeur du terroir, les relations avec l'extérieur, l'habitat et la répartition du peuplement.

Entre autres, les îles, comme de grandes prairies naturelles « flottantes » se prêtaient à une production agricole facile et abondante. Également, parce qu'il est situé au confluent du fleuve Saint-Laurent et de la rivière Richelieu, l'archipel du lac SaintPierre a joué un rôle stratégique pour la défense du territoire, un rôle militaire autant pour les autochtones Algonquins et les Iroquois, que pour les Français et plus tard, les Anglais.

L'établissement était défensif pour la colonie en Nouvelle-France ; les Anglais en ont aussi fait une plaque tournante pour la surveillance des Américains [...]. Ceux qui y venaient étaient réputés être de bons voyageurs, de bons marins [...]. Maintenant, le lac Saint-Pierre, le fleuve, les îles font partie de l'imaginaire collectif. Il n'y a pas de gens d'ici qui ne soient pas imprégnés de cette culture. (Lachapelle, 2004)

René Lachapelle (2004), animateur communautaire de la région, rapporte qu'à partir de la 2e guerre mondiale, de grandes industries de fabrication métallurgique, des chantiers navals et de fabrication de canons sont venus s'installer en bordure du lac Saint-Pierre. La récession d'après-guerre a été suivie d'un regain d'activités industrielles stimulé par la compétence des entreprises, la proximité d'abondantes sources de minerai et d'électricité. Le taux d'emplois manufacturiers était alors très élevé. La population a connu des années fastes sans précarité d'emploi. À partir des années 80 , l'industrie a commencé à péricliter, les pertes d'emplois massives ont plongé la région dans la pauvreté et la morosité. Plus récemment, afin de contrer cette situation, les décideurs ont mis l'accent sur la conversion industrielle et tentent de développer de nouveaux secteurs dans une démarche active et participative. Ce n'est pas chose facile, parce que la tradition d'entrepreneuriat a été délaissée, signale notre informateur.

9 Les titres de Réserve mondiale de la biosphère de l'UNESCO et de Site RAMSAR (Convention relative aux zones humides d'importance internationale) qu'a obtenus la région du lac Saint-Pierre témoignent de la richesse du milieu. Mais en même temps, ce site unique est bordé par des zones fortement industrialisées qui ont libéré 
d'importantes charges polluantes durant plusieurs décennies. La pollution diffuse à travers l'ensemble du bassin versant (agriculture, élevage, charges domestiques, etc.), l'érosion et la destruction d'habitats naturels ont également contribué à détériorer le milieu naturel. Même si la situation s'est nettement améliorée au cours de la dernière décennie, entre autres grâce aux efforts de plusieurs entreprises qui ont réduit leurs rejets, les contaminants rémanents et actuels, bioaccumulés par la faune (en particulier par le poisson et la sauvagine - principalement des oies et des canards migrateurs) peuvent poser un risque pour la qualité des écosystèmes et dans certains cas, pour la santé humaine. Par ailleurs, en certains endroits, la qualité bactériologique de l'eau est très mauvaise et ne permet aucun usage récréatif (Saint-Laurent Vision 2000, 2003). À ces problèmes régionaux d'ordre biophysique s'associent une situation de santé humaine peu enviable (telle que montrée, entre autres, par des indicateurs de santé comme le nombre de naissances de faibles poids, de problèmes cardiaques et de certains cancers) et des conditions de fragilité ou de précarité économique. Ainsi la région porte les marques du passage de grandes industries: pollution, paysages dévastés, économie chancelante.

\section{Contexte de recherche collaborative}

10 Bien que les communautés locales n'aient pas commandé ni participé (ou peu participé) à l'élaboration du projet COMERN ${ }^{2}$ et que, par conséquent, l'entreprise d'un tel projet exogène nous soit apparue a priori risquée, notre équipe de recherche en éducation relative à l'environnement a accepté d'y contribuer. Si, en effet, le problème de la contamination par le mercure n'est pas envisagé comme une préoccupation isolée, mais comme une porte d'entrée pour aborder l'ensemble du rapport à l'environnement, il devient alors possible de mieux légitimer l'invitation faite aux gens de la région à se joindre au projet, et de favoriser l'appropriation de ce dernier par les groupes sociaux concernés.

11 Par ailleurs, même s'il ne s'agit pas actuellement d'un problème prioritaire pour les gens du milieu, la contamination par le mercure les a toutefois déjà intéressés ou inquiétés dans un passé relativement récent (au cours des années '70 et ' 80 ). Le fait que des chercheurs étudient à nouveau le problème dans la région peut susciter de nouvelles craintes et dans ce contexte, un volet "éducation» accompagnant la démarche de COMERN apparaît encore plus pertinent : en particulier, il permet de faire le pont entre les chercheurs et les communautés en invitant celles-ci à y participer, à contribuer à une meilleure compréhension de la situation et à l'identification de solutions appropriées.

\section{Éléments de méthodologie}

Rappelons d'abord que notre étude de cas (débutée en 2001 et dont les activités se poursuivront jusqu'en 2006) est centrée sur deux principaux objets : d'une part, la relation des gens du Lac Saint-Pierre (à titre de région potentiellement «à risque »3) à la situation socio-environnementale de leur milieu, en lien avec leur santé, et plus spécifiquement en ce qui concerne la question du mercure: leurs représentations (incluant leurs attitudes, leurs valeurs, leurs savoirs, leurs croyances, leurs préoccupations, leurs attentes), leurs pratiques (leurs habitudes, la façon dont ils 
réagissent), leur motivation et leur capacité de participer à la prise en charge des problèmes, de même que les stratégies qu'ils envisagent ou déploient pour les résoudre; et d'autre part, la dynamique d'une éducation relative à l'environnement axée sur la santé environnementale, tel qu'elle parvient à s'installer au sein d'une démarche d'investigation collaborative visant la résolution de problèmes locaux et régionaux. Cette étude est menée à la fois dans une visée descriptive, explicative et heuristique (selon les catégories de Merriam, 1988 et de Yin, 1994) ${ }^{4}$. Nous avons adopté une posture épistémologique à la fois interprétative et critique (Robottom et Hart, 1993, in Sauvé, 1998-1999): au-delà de et à travers l'exploration de l'univers de significations des participants, il s'agit de favoriser le changement socioenvironnemental.

Pour cette étude de cas, intégrant recherche et intervention (recherche dans/par/pour l'intervention), notre méthodologie ${ }^{5}$ se caractérise par une approche collaborative (Desgagné, 2004), où notre rôle a été celui d'animer des échanges, des discussions et des travaux: nous avons invité des personnes clés d'organismes de la région (principalement dans les domaines de l'environnement, de la santé et du développement local), des groupes du milieu associatif (pêcheurs, femmes, citoyens) et aussi la communauté d'une institution scolaire (Cégep de Sorel-Tracy) à participer à une démarche d'investigation de la problématique de santé environnementale de la région (à partir du cas du mercure) et de recherche de solutions, incluant la mise au point de stratégies éducatives appropriées à chaque groupe ciblé.

Pour cette démarche, nous avons adopté les stratégies suivantes, servant autant les visées de recherche que celles qui concernent l'intervention : recherche documentaire, entretiens individuels formels et informels, entrevues de groupe (ou groupes de discussion), ateliers de travail collaboratif, forum et observation participante. Les données issues des divers échanges (saisies par enregistrement sonore ou vidéo) ont été transcrites sous forme de verbatims. Ces données, de même que les autres types de matériaux de recherche, ont fait l'objet d'une analyse qualitative. Les premiers résultats ont été validés auprès des participants lors d'un colloque/forum régional.

\section{Résultats et discussion}

15 Tel qu'annoncé, nous présentons dans cet article quelques résultats de la dimension phénoménologique de notre étude de cas auprès de/avec certains groupes et autres acteurs de la région. Nous tenterons de mettre en lumière certains aspects culturels de la situation étudiée sur ce territoire particulier du lac Saint-Pierre, afin d'y trouver un ancrage pour une éducation relative à la santé environnementale, qui soit appropriée au regard du contexte. Nous nous pencherons ici sur trois catégories de participants, dont la relation au territoire est contrastée : 1) les pêcheurs et autres «acteurs/ usagers » du lac, 2) les femmes et 3) les jeunes du milieu collégial, de même que leurs enseignants.

\section{Les pêcheurs sportifs et autres « acteurs/usagers " du lac ${ }^{6}$}

$16 \mathrm{Au}$ total, nous avons rejoint près de soixante personnes, citoyens rencontrés lors d'événements communautaires ou membres d'associations de la région du lac SaintPierre $^{7}$, qui de près ou de plus loin, dans leurs loisirs ou leur activité citoyenne, sont 
concernés par le lac, élément dominant de leur territoire. Voici quelques observations et éléments de réflexion issus de ces rencontres ${ }^{8}$.

Lorsqu'ils sont questionnés sur l'importance du lac Saint-Pierre pour eux-mêmes, des pêcheurs répondent : C'est ma raison de vivre! C'est notre lien à l'histoire, les parents de nos grands-parents viennent d'ici. C'est de l'air frais, le bien-être, le calme. C'est une richesse naturelle, un apport économique. Pour eux, le lac Saint-Pierre c'est aussi, comme le souligne David Orr (1992, p. 126, traduction libre) :

[...] davantage qu'un lieu, qu'une réalité géographique. C'est un laboratoire d'observation et d'expérimentation; une bibliothèque d'informations sur la géologie, l'histoire, la flore et la faune; c'est une source d'inspiration et de ressourcement ; c'est un terrain où l'on se mesure à soi-même.

18 Témoins importants des moindres changements du milieu, les pêcheurs connaissent bien les problèmes et les enjeux environnementaux au lac Saint-Pierre, tout comme les autres "usagers» du lac que nous avons interrogés. Les bas niveaux d'eau les inquiètent particulièrement. Il y a aussi les dommages causés par la navigation maritime et de plaisance, le dragage des sédiments, la surpêche, la diminution des populations de poissons, le braconnage, la présence d'obus au fond du lac (projectiles d'essai de la Défense nationale), la pollution industrielle et celle liée à l'épuration des eaux usées, l'introduction et la prolifération d'espèces envahissantes (moule zébrée, tanche, châtaigne d'eau) et la disparition d'espèces indigènes (crapet, bar rayé), la pollution diffuse liée à l'agriculture et l'expansion de l'« écotourisme ». Ainsi pour eux, la contamination par le mercure n'est que l'un des nombreux aspects d'une problématique globale. Ces "acteurs/usagers » ont vu se transformer le milieu. Ils se souviennent des années sombres de la pollution industrielle et urbaine des années 70 aux années 90. Leur perception de la qualité du milieu a également évolué : il y a 25 ans, la pollution ne soulevait que peu de craintes, mais aujourd'hui, ses diverses formes sont reconnues et dénoncées. Certains d'entre eux, travailleurs en industrie habitués à un environnement hostile, disent aujourd'hui : On ne tolère plus certaines situations acceptées auparavant. En usine, les règles de santé et sécurité sont très strictes et les comités de santé et sécurité sont très vigilants [...]. Les usines s'en font maintenant une gloire (Hélène Gignac, 2004). Les gens ont été sensibilisés et ont reconnu les dangers que posent les contaminants dans leur milieu de travail. Ils ont appris à faire le lien entre santé, travail et environnement. Alors aujourd'hui, plusieurs personnes ont modifié leurs pratiques, autant au niveau santé et sécurité qu'au niveau environnement, poursuit Hélène Gignac.

Volontaires pour l'étude du volet «santé » du projet COMERN (analyse des niveaux de mercure dans les cheveux et dans le sang), leur connaissance du milieu (relative à la dynamique hydrologique et celle des populations de poissons, ou encore à la commercialisation locale du poisson, par exemple) a permis d'orienter certaines décisions méthodologiques et certaines interprétations des chercheurs scientifiques. Les pêcheurs en particulier ont une expérience diachronique du lac, et sur toute son étendue ; ils en ont une expérience sensible bien particulière. Lors de rencontres avec les autres groupes communautaires participant au projet, les pêcheurs dits «sportifs ", comme les membres d'organisations à vocation de conservation, sont apparus comme des porteurs d'une certaine "sagesse environnementale ", des gardiens d'un territoire qu'ils habitent, qu'ils connaissent bien et qu'il faut protéger à tout prix. 


\section{Les femmes}

20 Nous avons choisi de rencontrer des femmes de la région pour explorer la question de la santé environnementale en lien avec la sécurité des aliments parce que l'intérêt des femmes pour la santé et la nutrition est généralement reconnu (en raison des rôles sociaux qu'elles assument bien souvent) et semble être associé à la sensibilité particulière qu'elles ont à l'égard de la vie. "Je surveille l'alimentation de mes trois filles, de mon mari, de mes amis [...]. Histoire de femme, quoi! La santé [...] passe nécessairement par l'assiette » (Lambert-Lagacé, 1988, p. 11). Ce réflexe de protection [...] est-il maternel, plus féminin? Il reste que les femmes prennent souvent en charge l'alimentation, le développement, l'éducation (Hélène Gignac, 2004).

21 Pour les femmes interrogées, membres d'associations ou personnes-clés du milieu (en environnement, santé ou développement local) ${ }^{9}$, le lac Saint-Pierre représente : La beauté, Notre chez-nous, Notre fierté. Chez elles, l'alimentation relie la famille au territoire. Dans certains cas, les populations «mangent littéralement leur environnement» (Payeur, 2001). La plupart des femmes signalent qu'une bonne nutrition est essentielle à une bonne santé, et celle-ci favorise à son tour un meilleur développement personnel. Cependant, elles constatent qu'il existe des inquiétudes et un manque d'information au sujet de la contamination des aliments, en particulier en ce qui concerne les besoins alimentaires des femmes à différentes étapes de leur vie, lors de la grossesse par exemple. Elles observent aussi un manque d'information quant à la provenance des aliments, leur origine environnementale et leur innocuité, critères pouvant les aider à faire un choix éclairé d'aliments sains pour elles-mêmes et leur famille: J'ai un doute concernant les recommandations de consommation de poissons [...]. L'information est incomplète. J'ai entendu beaucoup de choses négatives concernant la consommation poisson, surtout lorsque j'étais enceinte. Je trouve l'information alarmiste.

Les inquiétudes au sujet de la sécurité des aliments et de la santé les amènent à se questionner sur la qualité de l'environnement. À cet effet, les femmes reconnaissent qu'elles peuvent jouer un rôle important dans le maintien de la santé humaine en contribuant à la promotion de la santé des écosystèmes, étroitement liée à la qualité de vie. Notre projet de recherche-intervention devient pour elles une occasion intéressante de réfléchir aux enjeux de santé environnementale et de contribuer à amorcer un changement social. Il ne s'agit certes pas de promouvoir un écoféminisme de type psychobiologique, qui se base sur des arguments susceptibles de renforcer des stéréotypes comme celui qui associe la femme à la mère Nature (rôle passif et introverti, dont Janet Biehl, 1991, montre les limites), mais de faire valoir l'expérience et les savoirs des femmes contemporaines, leurs préoccupations, leur analyse des situations, leurs valeurs personnelles et sociales, situant le «caring» (l'attention soutenue et affectueuse) dans une perspective d'engagement social (à dimension politique également) et de recherche de solutions. À cet effet, au regard des situations de risque ou d'incertitude comme celle qui a trait au mercure et aux autres contaminants, les femmes interrogées mettent l'accent sur la communication à travers les réseaux locaux en place qui s'avèrent efficaces. 


\section{Les jeunes et leurs enseignants}

23 En milieu formel, au Cégep de Sorel-Tracy, près de 300 étudiants et douze enseignants et enseignantes ont participé à un processus de recherche-action pour l'intégration de l'éducation relative à l'environnement dans les curriculums de différents programmes, en particulier en Sciences de la nature, Sciences humaines, Technique infirmière, Technique de génie mécanique, Bureautique et enfin, Environnement, hygiène et sécurité au travail. Chaque groupe d'enseignant-étudiants a développé ou participé à un projet pédagogique axé sur l'exploration de la problématique des contaminants dans la région du lac Saint-Pierre ${ }^{10}$.

Force vive au sein de la société, les jeunes du collégial sont considérés comme d'importants acteurs de changements dont la formation actuelle contribuera à façonner leur vision du monde. La jeunesse, la créativité et l'énergie sont porteuses d'espoir chez cette génération appelée à construire une citoyenneté responsable. De plus, les jeunes peuvent rejoindre divers groupes au sein de leur communauté (famille, amis, entourage, etc.) et ainsi contribuer à insuffler autour d'eux une dynamique de changement de mentalités et de conduites. Les étudiants peuvent également contribuer à la définition de la réalité socio-environnementale régionale telle qu'ils la perçoivent, ainsi qu'à l'identification de solutions aux problèmes qu'ils identifient comme prioritaires. Leur étude et leur analyse de la problématique de la contamination, de même que leurs réflexions critiques ont été présentées lors d'expositions et lors d'un événement régional (Journée Complices en Environnement) ouvert au grand public (Godmaire et coll., 2004). Les jeunes participants ont été appréciés comme " une relève prometteuse, un espoir ». Mais il faut reconnaître que, de façon générale, chez les jeunes, l'ancrage au milieu est faible ou précaire.

La perception qu'ont les jeunes du lac Saint-Pierre est différente de celle des générations précédentes. À partir du moment où l'accès à l'eau a été réduit par l'établissement des usines et des chantiers navals en bordure du lac et des affluents, les jeunes n'ont pas pu développer le même rapport au milieu aquatique que leurs aînés. Moi quand j'étais jeune, on allait marcher jusqu'au bout du quai puis après on marchait sur la grève pour aller ramasser des grenouilles... ce n'est plus possible aujourd'hui (René Lachapelle, 2004). Puis, il y a eu la privatisation des berges et l'étalement urbain qui ont accentué le phénomène. L'image qu'ont les jeunes de la région est construite à partir des médias et de quelques enseignements scolaires. Rares sont ceux qui ont eu l'occasion jusqu'ici de vivre des expériences concrètes de "rencontre » avec le lac, de profiter par exemple de son «potentiel récréo-touristique». De façon générale, à travers les projets pédagogiques auxquels ils ont participé dans le cadre de cette recherche-intervention, les étudiants ont apprécié leur exploration des réalités du lac Saint-Pierre : J'ai aimé en apprendre davantage sur la situation de notre région étant donné que nous étions plus ou moins informés. J'ai apprécié le projet, car il nous touche directement: le lac Saint-Pierre nous appartient.

Un enseignant participant au projet témoigne de la nécessité d'explorer et comprendre son milieu de vie pour construire sa propre identité :

Il faut s'approprier, nommer les choses, s'approprier le lieu, en faire une fierté, le respecter. Malheureusement, on observe actuellement une rupture entre le temps et le lieu. Il y a un certain malaise dans la région, on n'a qu'à penser aux nombreux suicides au cours des dernières années, entre autres chez les étudiants du collégial. Le lieu, le lac Saint-Pierre, est omniprésent, il faut davantage « donner du lieu », car 
le lieu a un potentiel catalyseur, il peut devenir un projet de vie qui nous porte. Le lieu peut également servir de lien intergénérationnel. À défaut d'avoir une appartenance plus vaste, il est possible d'avoir une appartenance de proximité. L'éducation permet une meilleure compréhension du lieu qu'on habite. Elle est à faire au Cégep, mais aussi ailleurs, au-delà. La communauté devrait se donner un projet collectif en solidarité sur une question socio-environnementale. Cela donnerait un sens à la vie. (Yves Saint-Sauveur, 2003) particulier au territoire. Chacun appréhende de façon particulière la relation entre son environnement et la santé. Chacun s'imprègne et participe de façon différente à la "culture régionale». Pour les pêcheurs par exemple, le lac, élément dominant du territoire, est un lieu d'être et d'actualisation, de relation avec la nature, un patrimoine à préserver. Pour les femmes, le territoire est un milieu de vie, source d'alimentation et de santé familiale, domestique. Pour les jeunes, la signification du territoire est à construire : c'est un lieu de découverte, d'apprentissage, une perspective d'ancrage à la vie elle-même. Et ces diverses façons d'appréhender le territoire sont certes des points 
de départ différents pour concevoir des projets d'éducation relative à l'environnement qui soient appropriés à chacun des contextes.

Mais à travers ces différences, certaines constantes sont apparues. Lorsqu'on a initialement abordé avec les participants la question des contaminants, en particulier celle du mercure dans le poisson, ces derniers ont d'emblée ouvert la discussion sur une problématique beaucoup plus vaste ; ils ont fait «éclater " le problème en le recadrant dans une perspective écosystémique. Par ailleurs, tous ont établi clairement le lien entre la santé humaine et celle de l'environnement: la qualité de l'eau, de l'air, des aliments du terroir (et d'ailleurs) a un impact sur notre santé. Aussi, de façon générale, les participants se questionnent sur la qualité (innocuité et salubrité) non seulement du poisson du lac Saint-Pierre, mais de celle du poisson, des fruits de mer et de tous les aliments de toutes provenances: Doit-on réduire sa consommation de poisson? Où peut-on trouver des recommandations fiables? Quelle est la qualité des produits du supermarché ? Ce n'est pas en effet la question du mercure qui préoccupe d'abord, mais plutôt celle de l'ensemble des contaminants dans tous les aliments :

Tout est pollué, la viande aussi, la vache, l'herbe qu'elle broute dans son champ. Je suis inquiet et craintif. J'ai peur en particulier des pesticides. La pollution tombe par terre et les animaux la mangent. On dit qu'elle tombe sur les légumes, dans l'herbe, le foin. Je mange bien plus de viande animale terrestre que de poisson [...] ma nourriture est polluée. Connaitt-on les effets des polluants de l'environnement sur les humains ? Y a-t-il un problème avec les métaux dont les concentrations sont élevées dans le fleuve?

Ces inquiétudes semblent partagées. Comme le rapporte Hubert (2002, p. 9 et 103), les modes de production alimentaire actuels font en sorte qu'on a perdu contact avec des choses fondamentales comme la provenance et la nature de la nourriture qu'on consomme :

Manger est à la fois un acte biologique et culturel. Si le « mangeur » perd le contact, la trace de ses aliments, il ressent un malaise, d'où le désir et le besoin de renouer avec toute la chaîne alimentaire : de la fourche à la fourchette! [...] Vache folle, listéria, fièvre aphteuse, nitrates, OGM, pesticides [...] Notre alimentation met-elle en péril notre santé ? Sommes-nous mal informés ? Trop informés?

Les attitudes des gens face au risque que peut entraîner la consommation de poisson du lac sont variées, confirmant ainsi les catégories de réactions relatives au risque identifiées par Larue et coll. (1997). Certaines personnes ont abandonné la consommation de ce poisson parce qu'elles considèrent que le milieu est trop pollué ou encore parce qu'elles ont reçu ou saisi dans le passé des informations inquiétantes. D'autres participants adoptent une consommation modérée, qu'ils jugent sécuritaire : Je fais attention, mais j'en mange quand même. Pour d'autres, il n'y a pas lieu de s'inquiéter : [...] la situation n'est pas pire ici qu'ailleurs. D'après ce que j'ai entendu, le poisson mis en marché commercialement est aussi contaminé que le poisson pêché au lac Saint-Pierre.

De façon générale, les participants déplorent le manque d'information adéquate en provenance du réseau des organismes de santé publique. Entre autres, ils observent des contradictions. Dans certains cas, ils dénoncent le caractère alarmiste des informations véhiculées en ce qui concerne les impacts de la consommation de poisson sur la santé humaine. Ils expriment le besoin d'une information cohérente, continue, mise à jour et qui intègre des explications physiologiques des impacts d'une exposition au mercure (ou autre contaminant) par la consommation de poisson. Ils soulignent toutefois 
l'importance de ne pas inquiéter inutilement la population : il faut faire comprendre la situation, mais il ne faut pas faire peur. Il faut aussi soulever le questionnement. Enfin, il faut se poser la question : socialement, qu'est-ce qu'on fait?

Parmi les solutions envisagées par les participants, on suggère que la qualité du poisson du lac soit rigoureusement contrôlée; qu'un meilleur suivi de l'activité de pêche soit effectué afin d'éviter la diminution des populations de poisson; que le poisson d'ici et d'ailleurs soit étiqueté (provenance et teneur en contaminants) ; que l'information soit disponible aux comptoirs des marchés d'alimentation.

Satisfaire nos besoins de traçabilité est aussi une manière de nous tranquilliser et d'endiguer la panique. Ce besoin de renouer une communication plus directe avec nos aliments est un besoin fondamental (de l'ordre de la sécurité alimentaire). Ce lien que la plupart d'entre nous ont perdu, et ce parfois depuis plusieurs générations, n'est pas uniquement de l'ordre du souci sanitaire, mais une nécessité affective et de communication. Cela tient surtout de l'imaginaire et de l'immatériel, mais il est important de le satisfaire pour une meilleure qualité de vie. Les " crises " alimentaires n'ont fait qu'exacerber le besoin de renouer un lien direct entre soi et ses nourritures. Savoir d'où elles viennent, comment elles sont produites et par qui. (Hubert, 2002, p. 103)

De façon générale, les échanges avec/entre les participants ont mis en évidence la complexité de la problématique de la contamination : l'état de détérioration du lac est la conséquence d'un ensemble de facteurs, associés à une multitude d'acteurs et de pratiques. Les instances législatives, les industries, les municipalités, les usagers du lac (armateurs et plaisanciers) et les riverains, tous sont identifiés comme ayant une part de responsabilité. L'éventail des solutions envisagées reflète la multiplicité des causes et la diversité des acteurs concernés. Plus spécifiquement, les femmes insistent sur l'importance de construire ou de mettre à profit un réseau de communication efficace ; les jeunes mettent l'accent sur l'engagement dans des projets d'action; les pêcheurs souhaitent faire valoir leurs connaissances et leur rôle de " gardiens du milieu ».

\section{Des stratégies éducatives appropriées}

Nous sommes maintenant à l'étape de concevoir avec des collaborateurs des différents groupes sociaux concernés, des stratégies éducatives appropriées à chacune des populations cibles. La mise au jour des savoirs, des représentations et des attentes de chacune de ces dernières, de sa relation particulière au territoire et de sa culture de référence offre un diagnostic essentiel au démarrage d'une telle tâche. Par ailleurs, l'exploration de différents courants d'intervention en éducation relative à l'environnement (Sauvé, 2003) nous a permis de repérer deux approches particulièrement pertinentes au regard des problématiques socioécologiques en question: celle du biorégionalisme et celle de la critique sociale. L'approche biorégionale (Traina et Darley-Hill, 1995; Pruneau et coll., 1997) est axée sur la reconstruction du lien d'appartenance au milieu; elle mise sur la valorisation de la culture et des talents des gens, de même que sur les caractéristiques et les potentialités $\mathrm{du}$ milieu biophysique pour contribuer à un développement local, endogène et responsable. L'approche de la critique sociale (Fien, 1993 ; Robottom et Hart, 1993) met l'accent sur l'investigation collaborative et critique des réalités socioécologiques dans une perspective de construction de savoirs pertinents au regard du contexte et de la transformation des réalités qui posent problème. À travers cette action sociale, c'est en 
fin de compte la transformation de ses acteurs qui est visée, vers plus d'autonomie et de pouvoir-faire.

C'est donc sur la trame de fond de ces approches complémentaires et en fonction d'une conception holistique de la santé environnementale (Sauvé et Godmaire, 2004) que nous avons entrepris une démarche collaborative de conception et d'expérimentation de stratégies éducatives, plus spécifiquement auprès des pêcheurs et des femmes de la région. Nous poursuivons également en milieu collégial la mise au point et/ou la modélisation des pratiques d'éducation relative à l'environnement auprès des jeunes, qui se sont avérées plus porteuses en première phase de recherche.

Mais déjà, la première étape de ce projet nous a permis de vérifier le potentiel éducatif et mobilisateur d'une démarche d'exploration collective de la dimension psychosociale de la problématique. Le processus de questionnement et de réflexion à travers les entretiens, les discussions de groupe et les ateliers de travail a permis aux participants de mieux se situer eux-mêmes au regard de la problématique abordée, d'échanger entre eux des informations, des observations, différentes façons de voir les choses, de rechercher des informations manquantes ou d'en valider d'autres. Cette entrevue me fait vraiment réfléchir. Je prends conscience des raisons "pas toujours rationnelles» de mes choix alimentaires. C'est très éducatif. Le processus a permis d'éveiller l'intérêt pour les questions abordées, condition première d'engagement. En tant que conseiller municipal, je m'engage, avec l'appui de ma ville, à solliciter toutes les municipalités riveraines pour l'appui et la poursuite du projet. L'un des points forts de notre démarche a été de créer des occasions de rencontre (échange et réflexion) entre des acteurs du milieu de l'éducation formelle et du milieu associatif, des citoyens, des décideurs et des chercheurs. Faire converger les visions du territoire, les savoirs, les inquiétudes et les désirs fut un exercice enrichissant que les participants désirent répéter, dans la perspective de mettre à profit la complémentarité des apports de chacun et la nécessité de renforcer la culture de concertation déjà présente dans le milieu. Au bilan, cette démarche implicite d'éducation relative à l'environnement aura contribué à donner un « espace » de réflexion, des mots, des idées, des stratégies et des outils aux participants. Ces derniers souhaitent que la complicité amorcée entre eux se poursuive, stimule et soutienne un engagement dans la prise en charge collective des réalités socioécologiques de leur territoire partagé.

\section{BIBLIOGRAPHIE}

Biehl, J. (1991). Finding Our Way - Rethinking Ecofeminist Politics. Montréal : Black Rose Books.

Corcoran, P.B., Walker, K.E. et Wals, A.J. (2004). Case studies, make-your-case studies, and case stories : a critique of case study methodology in sustainability in higher education. Environmental Education Research, 10(1), 7-22.

De Konink, R. (2000). Les cent îles du lac Saint-Pierre. Retour aux sources et nouveaux enjeux. SainteFoy : Les Presses de l'Université Laval. 
Desgagné, S. (2004). La participation de l'usager, « praticien » ou « citoyen », à la production d'un savoir crédible : une avenue incontournable. Communication présentée au Colloque « Le croisement des savoirs au cœur des recherches en éducation relative à l'environnement : épistémologies, méthodologies, enjeux et défis ", ACFAS mai 2004, Chaire de recherche du Canada en éducation relative à l'environnement.

Feagin, J., Orum, A. et Sjoberg, G. (1991). A case for case studies. Chapel Hill : University of North Carolina Press.

Fien, J. (1993). Education for the environment. Critical curriculum theorising and environmental education. Deakin (Victoria) : Deakin University Press.

Gignac, H. (2004). Entretien de recherche collaborative. Document non publié dans le cadre du projet COMERN.

Godmaire, H., Bonin, P. et Sauvé, L. (2004). Journée « Complices en environnement ». Actes. Montréal : Les Publications ERE-UQAM.

Hubert, A. (2002). Pas de panique! (alimentaire). Paris : Marabout.

Lachapelle, R. (2004). Entretien de recherche collaborative. Document non publié dans le cadre du projet COMERN

Lambert-Lagacé L. (1988). Le défi alimentaire. Montréal : Les Éditions de l'Homme.

Larue, R., Fortin, J. et Michard, J.L. (2000). École et santé : le pari de l'éducation. Paris : Hachette.

Larue, R., Laliberté, C. et Grondin, J. (1997). Guide de consommation de pêche sportive en eau douce: une évaluation. Longueuil : Direction de la santé publique de la Montérégie et Centre de santé publique de Québec.

Laverdière, C.H. (1870). Oeuvre de Champlain (2e édition). Sainte-Foy : Université Laval.

Lebel, J. (2003). La santé, une approche écosystémique. Ottawa : Centre de recherches pour le développement international (CRDI).

Merriam, S. (1988). Case Study in Education : A Qualitative Approach. San Francisco : Jossey-Bass.

Orr, D. (1992). Ecological Literacy - Education and the transition to a postmodern World. Albany : State University of New York Press.

Payeur, S. (2001). La santé publique : l'affaire d'une société. Découvrir, 22(5), 44-52.

Pruneau, D., Breau, N. et Chouinard, O. (1997). Un modèle d'éducation relative à l'environnement visant à modifier la représentation des écosystèmes biorégionaux. Éducation et francophonie, Revue scientifique virtuelle, 25(1), consulté le 22 octobre 2002 sur http://www.acelf.ca/revue/XXV1/ articles/.

Robottom, I. et Hart, P. (1993). Research in Environmental Education. Engaging the Debate. Deakin (Victoria) : Deakin University Press.

Saint-Laurent Vision 2000. (2003). Portrait global de l'état du Saint-Laurent. Consulté le 13 novembre 2003 sur http://www.slv2000.qc.ca/plan_action/phase3/biodiversite/.

Santé Canada. (1999). Guide canadien d'évaluation des incidences sur la santé. Volume 1 : Notions fondamentales. Consulté le 12 février 2003 sur http://www.hc-sc.gc.ca/ehp/dhm/behm/eis/ vol1.htm.

Sauvé, L. (1998-1999). Un patrimoine de recherche en construction. Éducation relative à

l'environnement : Regards - Recherches - Réflexions, 1, 13-40. 
Sauvé, L. (2003). Courants et modèles d'interventions en éducation relative à l'environnement. Module 5. Programme d'études supérieures - Formation en éducation relative à l'environnement Francophonie internationale. Université du Québec à Montréal - Collectif ERE-Francophonie. Montréal : Les Publications ERE-UQAM.

Sauvé, L. et Godmaire, H. (2004). Environmental Health Education : A theoretical and praxical proposal. Ecohealth (CRDI), Déc. 2004, 35-46.

Sauvé L. et Godmaire, H. (2005). L'éducation relative à la santé environnementale : une dimension intégrante du projet COMERN. Rapport de recherche-intervention. (Document interne en production continue).

St-Sauveur, Y. (2003). Entretien de recherche collaborative. Document non publié dans le cadre du projet COMERN.

Traina, F. et Darley-Hill, S. (1995). Perspectives in Bioregional Education. Troy (OH) : NAAEE.

Villedieu, Y., (2002). Un jour la santé. Montréal : Édition Boréal.

Yin, R.K. (1994). Case Study Research : Design and Methods. (2e édition). Beverly Hills : Sage Publishing.

\section{NOTES}

1. Pour plus d'informations, consulter le site Internet suivant: http://www.unites.uqam.ca/ comern.

2. Ce projet, qui adopte des approches interdisciplinaire, écosystémique et participative, comporte trois principaux volets : un volet de recherche biogéophysique, un volet de recherche en sciences de la santé et un volet de recherche-intervention en éducation.

3. Les travaux réalisés depuis 2001 dans le cadre des volets biogéophysique et « santé » du projet COMERN ont montré qu'en ce qui concerne plus spécifiquement le mercure, les concentrations présentes dans le lac ne sont pas actuellement inquiétantes. La recherche se poursuit entre autres sur les effets synergiques des contaminants entre eux.

4. Dans une visée descriptive, il s'agit de saisir, comme nous l'avons signalé, " un système culturel en action " de façon à développer la connaissance (à travers ce cas particulier et les savoirs transférables qui en résultent) du phénomène de la relation à la santé environnementale des gens d'une région «à risque » ainsi que des conditions et processus d'une éducation relative à la santé environnementale adoptant un processus participatif. Dans une visée explicative, il s'agit de vérifier l'impact d'une culture régionale particulière et l'influence d'autres facteurs (identifiés en cours de route) sur les phénomènes et processus en question. Dans une visée heuristique, l'étude de cas mène à l'émergence de nouvelles questions de recherche et d'hypothèses et aussi à l'idéation de projets d'intervention éducative et de solutions socio-environnementales appropriées.

5. La méthodologie détaillée de cette recherche fait l'objet d'un rapport en production continue (Sauvé et Godmaire, 2005).

6. Nous empruntons cette idée d'« acteurs " à la nouvelle Politique de l'eau du Québec, axée sur la gestion par bassins versants et qui invite les citoyens à se considérer comme les «acteurs de l'eau ».

7. Principalement, nous avons travaillé avec les organisations suivantes : le Comité de la zone d'intervention prioritaire du lac Saint-Pierre, l'Association de chasseurs et pêcheurs de SainteAnne de Sorel, la Table de concertation en environnement du Bas-Richelieu, la Corporation de développement communautaire de Nicolet-Yamaska. 
8. Valérie Lacourse, étudiante à la maîtrise en sciences de l'environnement de l'UQAM (Université du Québec à Montréal), a également contribué à la réalisation et la transcription des entrevues auprès des pêcheurs et des femmes.

9. Plus spécifiquement, nous avons rencontré les femmes du Groupe l'Héritage de Louiseville et de la Corporation de développement communautaire de Nicolet-Yamaska.

10. Cette partie du notre étude de cas régionale a été menée en étroite collaboration avec Patrick Bonin, étudiant à la maîtrise en sciences de l'environnement de l'UQAM, qui en a fait l'objet de son mémoire de maîtrise (2006): Enjeux et stratégies d'éducation relative à l'environnement en milieu collégial. Le cas du Cégep de Sorel-Tracy au regard de la problématique des contaminants dans la région du lac Saint-Pierre.

\section{RÉSUMÉS}

Dans le contexte d'un vaste projet de recherche écosystémique et interdisciplinaire sur la question de la contamination par le mercure, nous avons invité des membres de diverses communautés de la région du Lac Saint-Pierre (Québec) à participer à l'exploration et à la résolution de cette problématique. Il importait en effet de considérer la dimension socioculturelle de cette dernière: au lac Saint-Pierre, en lien avec les caractéristiques particulières de ce territoire d'une très riche biodiversité, il existe une culture régionale spécifique, ancrée dans la relation au milieu et dans les pratiques ancestrales de chasse et de pêche, qui « colore » fortement la situation socio-environnementale en question. Notre travail a donc consisté à mettre en place une dynamique d'échange, de discussion et de travail collaboratif, plus spécifiquement avec des groupes de pêcheurs, de femmes et de jeunes, de façon à clarifier entre autres les préoccupations, les besoins, les savoirs et les pratiques des participants concernant la problématique de la contamination du lac et de la région. Ces informations enrichissent certes la démarche de recherche du projet scientifique, mais plus encore, le processus qui les génère porte en lui-même un fort potentiel d'éducation communautaire et de mobilisation des groupes concernés pour la prise en charge des réalités de leur milieu de vie.

In the context of a vast ecosystemic and interdisciplinary research project on mercury contamination, we invited members of various communities of the Lake Saint-Pierre region (Québec) to participate in the exploration and resolution of this issue. It was indeed important to take into account the socio-cultural dimension of the latter : in the Lake Saint- Pierre region, in relation with the specific characteristics of this highly biodiversified area, there is a specific regional culture, anchored in the relation to the environment and the ancestral practices of hunting and fishing, which strongly influences the socio-environmental issue under consideration. Our work thus consisted in setting up a collaborative discussion and investigation process, more specifically with groups of fishermen, women and youngsters, so as to clarify among others their concerns, needs, knowledge and practices related to the contamination issue in the Lake and region. The information collected obviously enriches the scientific project's research approach, but moreover, the collaborative process itself shows a strong potential for community education and the mobilization of the concerned groups for their empowerment in relation to the realities of their environment. 


\section{AUTEURS}

\section{HÉLÈNE GODMAIRE}

Titulaire d'une maîtrise et d'un doctorat en biologie aquatique, elle est également diplômée du programme court de 2e cycle en éducation relative à l'environnement à l'UQAM. Dans le cadre de la Chaire de recherche du Canada en éducation relative à l'environnement, elle coordonne actuellement le volet d'éducation relative à l'environnement du projet COMERN (Collaborative Research Network Program on the Impacts of Atmospheric Mercury Deposition on Large Scale Ecosystems in Canada).

\section{LUCIE SAUVÉ}

Professeure au département d'éducation et de pédagogie de l'Université du Québec à Montréal, elle est titulaire, au sein de cette institution, de la Chaire de recherche du Canada en éducation relative à l'environnement, membre de l'Institut des sciences de l'environnement et de l'Institut santé et société. Elle est aussi directrice du Programme d'études supérieures en éducation relative à l'environnement (ERE). Elle dirige des projets de recherche principalement axés sur l'institutionnalisation de l'ERE en milieu scolaire, sur la formation des enseignants et autres éducateurs, et sur l'intervention en milieu communautaire, tant au Québec qu'en contexte de coopération internationale. Elle est responsable du projet ERE-Mercure, dans le cadre du réseau COMERN. 